\title{
High grade glioma and caregiver burden
}

\author{
David O'Keeffe ${ }^{1} \cdot$ Richard M. Bambury ${ }^{2} \cdot$ Seamus O'Reilly ${ }^{2}$
}

Received: 26 February 2021 / Accepted: 29 March 2021 / Published online: 15 April 2021

(c) The Author(s), under exclusive licence to Springer Science+Business Media, LLC, part of Springer Nature 2021

\section{To the Editor}

We noted with interest the article by Sharma et al. on the longitudinal perception of prognosis in newly-diagnosed high grade glioma patients and caregivers compared to clinicians [1]. Their study emphasises the significant detrimental effect of a glioma diagnosis on the caregiver - a burden that in our clinical practice seems disproportionate to that experienced with other cancer diagnosis and is probably related to illness associated cognitive decline and its associated impact on burden of care. Similar to Sharma et al. we have assessed this in a study of 26 patients and their caregivers in a questionnaire based study and noted that caregivers felt more worn out by illness and reported higher levels of psychological pain compared to patients, while both groups noted increasing levels of frustration related to uncertainty of the illness trajectory. Other groups have highlighted caregiver distress, with anxiety levels reaching the threshold for professional help in all caregivers at the time of brain tumor recurrence [2].

The caregiver burden highlighted in these studies persists beyond end of life care. A study by Fechl et al. surveyed 52 caregivers of patients with brain tumors a median of 3 years after the patient's death [3]. They noted poor quality of life regardless of whether the patient died in hospital or at home, a $29 \%$ incidence of financial difficulties with associated burnout in $60 \%$ of those affected, and that $29 \%$ of caregivers were incompletely prepared. Traditional oncology supports have focused significantly on the patient-these studies would suggest that for neurooncology units an enhanced carer focused pathway is also needed. In this regard the establishment of a randomised trial (German Register for Clinical Studies (DRKS)DRKS00016066)) evaluating the impact of early palliative care for patients with gliomas which includes assessment of caregiver burden is welcome. In the interim we feel that the study by Sharma et al. reinforces the need for greater awareness and more formalised support structures for caregivers in neurooncology practices.

\section{References}

1. Sharma A et al (2021) How much time do we have? Longitudinal perception of prognosis in newly diagnosed high grade glioma patients and caregivers compared to clinicians. J Neurooncol. https://doi.org/10.1007/s11060-021-03700-2

2. Trad W et al (2015) Screening for psychological distress in adult primary brain tumor patients and caregivers: considerations for cancer care coordination. Front Oncol. https://doi.org/10.3389/ fonc. 2015.00203

3. Flechl B et al (2013) The caregivers' perspective on the end-of-life phase of glioblastoma patients. J Neurooncol 112(3):403-411

Publisher's Note Springer Nature remains neutral with regard to jurisdictional claims in published maps and institutional affiliations.

\section{Letter to Editor in relation to: Sharma A et al. How much} time do we have? Longitudinal perception of prognosis in newly diagnosed high grade glioma patients and caregivers compared to clinicians. J Neurooncol Jan 23 https://doi.org/10.1007/s11060021-03700-2

Seamus O'Reilly

seamus.oreilly@hse.ie

School of Medicine, University College Cork, Cork, Ireland

2 Department of Medical Oncology, Cork University Hospital, Cork, Ireland 